F. Bagemihl

Nagoya, Math. J.

Vol. 53 (1974), 137-140

\title{
THE THREE-ARC AND THREE-SEPARATED-ARC PROPERTIES OF MEROMORPHIC FUNCTIONS
}

\author{
FREDERICK BAGEMIHL
}

Let $\Gamma$ be the unit circle and $D$ be the open unit disk in the complex plane, and denote the Riemann sphere by $\Omega$. Suppose that $f(z)$ is a meromorphic function in $D$, and that $\zeta \in \Gamma$. The principal cluster set of $f$ at $\zeta$ is the set

$$
\Pi(f, \zeta)=\bigcap_{A} C_{A}(f, \zeta)
$$

where $\Lambda$ ranges over all arcs at $\zeta$; the chordal principal cluster set of $f$ at $\zeta$ is the set

$$
\Pi_{x}(f, \zeta)=\bigcap_{x} C_{X}(f, \zeta),
$$

where $X$ ranges over all chords at $\zeta$; and we define the set

$$
\Delta(f, \zeta)=\Pi_{x}(f, \zeta)-\Pi(f, \zeta),
$$

where it is clear that $\Pi(f, \zeta) \subseteq \Pi_{x}(f, \zeta)$. We say that $f$ has the threearc property at $\zeta$, if there exist three $\operatorname{arcs} \Lambda_{1}, \Lambda_{2}, \Lambda_{3}$ at $\zeta$ such that

$$
C_{A_{1}}(f, \zeta) \cap C_{A_{2}}(f, \zeta) \cap C_{A_{3}}(f, \zeta)=\phi ;
$$

if, moreover, the three arcs can be taken to be mutually exclusive, we say that $f$ has the three-separated-arc property at $\zeta$; and if the three arcs can be taken to be chords at $\zeta$, we say that $f$ has the three-chord property at $\zeta$.

Gresser [5, p. 145, Theorem 2] has shown that there exists a meromorphic function in $D$ that has the three-chord property (and hence the three-separated-arc property) at each point of a perfect subset of $\Gamma$. Belna [4, p. 220] has raised the question of the existence of a meromorphic function in $D$ that has the three-separated-arc property at each point of $\Gamma$.

Received May 24, 1973. 
THEOREM 1. There exists a normal meromorphic function $f(z)$ in $D$ that has the three-separated-arc property at every point of $\Gamma$.

Proof. Let $f(z)$ be a Schwarzian triangle-function in $D$ whose fundamental triangle has angles $\pi / 7, \pi / 3, \pi / 2$, and let its system of triangles be that displayed in [6, p. 437, Fig. 122]. A vertex of the figure is a common vertex of either fourteen, six, or four of the figure's triangles; we assume that $f$ has the value $\infty, 0,1$ at a vertex of the first, second, third kind, respectively. Then, as is well known, $f$ is meromorphic and normal in $D$.

By a 14-star, 6-star, 4-star of the figure, we shall mean the union of the fourteen, six, four triangles having a common vertex of the first, second, third kind, respectively, which vertex will be called the center of the $n$-star; we consider the interior points as well as the boundary points as belonging to the triangle in question. The hyperbolic diameter of an $n$-star is independent of its center, and will be denoted by $d_{n}$ $(n=14,6,4)$. As $z$ describes the frontier of an $n$-star, $f(z)$ varies over the closed interval $I_{1}$ of the real axis between 0 and 1 if $n=14$, the closed interval $I_{2}$ of the positive real axis between 1 and $\infty$ if $n=6$, and the closed interval $I_{3}$ of the nonpositive real axis between 0 and $\infty$ if $n=4$.

Now suppose that $\zeta$ is any point of $\Gamma$. Denote the radius at $\zeta$ by $R$ and the diameter with end points $\zeta$ and $-\zeta$ by $M$. Let $G_{1}$ be the lens-shaped region between two hypercycles $H_{2}$ and $H_{3}$ through $\zeta$ and $-\zeta$, one on either side of $M$ and each the same hyperbolic distance greater than $d_{14}$ from $M$. Denote by $G_{2}, G_{3}$ the remaining two regions into which the hypercycles divide $D$, where $H_{2}, H_{3}$ is part of the frontier of $G_{2}, G_{3}$, respectively. Take a hypercycle $H_{2}^{\prime}$ in $G_{2}$ through $\zeta$ and $-\zeta$ such that its hyperbolic distance from $H_{2}$ is greater than $d_{6}$, and let $H_{3}^{\prime}$ be a hypercycle in $G_{3}$ through $\zeta$ and $-\zeta$ such that its hyperbolic distance from $H_{3}$ is greater than $d_{4}$.

Consider the union $U_{1}$ of those 14-stars that intersect the radius $R$ at $\zeta$, the union $U_{2}$ of those 6-stars that intersect the arc at $\zeta$ that extends along $H_{2}^{\prime}$ from some point of $H_{2}^{\prime}$ to $\zeta$, and the union $U_{3}$ of those 4-stars that intersect the arc at $\zeta$ that extends along $H_{3}^{\prime}$ from some point of $H_{3}^{\prime}$ to $\zeta$. For each (fixed) $n=14,6,4$, it is evident that $D$ is the union of all the $n$-stars of the figure, and any two of these $n$-stars are 
either mutually exclusive or have only frontier points in common. Consequently each of the sets $U_{1}, U_{2}, U_{3}$ is connected, and there are arcs $\Lambda_{1}, \Lambda_{2}, \Lambda_{3}$ at $\zeta$ which lie on the frontiers of the respective sets $U_{1}, U_{2}, U_{3}$. Since $U_{j} \subset G_{j}(j=1,2,3)$, the arcs $\Lambda_{1}, \Lambda_{2}, \Lambda_{3}$ are mutually exclusive. Moreover $C_{A_{j}}(f, \zeta) \subseteq I_{j}(j=1,2,3)$, and so $f$ has the three-separated-arc property at $\zeta$. In view of the fact that $\zeta$ was an arbitrarily chosen point of $\Gamma$, the proof of the theorem is complete.

Although there exists [3, p. 31, Theorem 4] a function of bounded characteristic in $D$ that has the three-arc property at each point of a perfect subset of $\Gamma$, the general behavior of a function of bounded characteristic relative to the three-arc property is markedly different from that of a normal function.

THEOREM 2. The set of points on $\Gamma$ at which a meromorphic function $f(z)$ of bounded characteristic in $D$ has the three-arc property is of measure zero.

Proof. According to [7, p. 208], $f$ has a radial limit at almost every point of $\Gamma$. If $\zeta \in \Gamma$, and if $f$ has the radial limit $\omega$ at $\zeta$, then unless $\zeta$ is an ambiguous point of $f$, we have $\omega \in C_{A}(f, \zeta)$ for every arc $A$ at $\zeta$, and consequently $f$ cannot have the three-arc property at $\zeta$. Since $f$ has at most enumerably many ambiguous points on $\Gamma[1$, p. 380 , Theorem 2], the theorem is proved.

The following question was raised in [3, p. 32, Question 4]:

"Let $f$ be of class $(A)$ in $D$; that is, let $f$ be bounded and holomorphic in $D$, and let the radial limit of $f$ have modulus 1 at almost all points $e^{i \theta}$. Does $f$ have the three-arc property at all (or almost all) singular points $e^{i \theta}$ that are not isolated singularities?"

Theorem 2 answers this question in the negative, because if we take $f$ to be a Blaschke product such that the set of limit points of its zeros is $\Gamma$, then every point of $\Gamma$ is a nonisolated singularity of $f$, but $f$ has the three-arc property almost nowhere on $\Gamma$.

There exists [3, p. 33] a normal holomorphic function $f$ in $D$ such that $\Delta(f, \zeta)=\Omega$ for almost all $\zeta \in \Gamma$; and there exists [2] a meromorphic function $f$ in $D$ such that $\Delta(f, \zeta)=\Omega$ for all $\zeta \in \Gamma$. The proof of Theorem 2 shows, however, that for functions of bounded characteristic we have the following 
COROLLARY. If $f(z)$ is a meromorphic function of bounded characteristic in $D$, then $\Delta(f, \zeta)=\phi$ for almost all $\zeta \in \Gamma$.

\section{REFERENCES}

[1] F. Bagemihl, Curvilinear cluster sets of arbitrary functions, Proc. Nat. Acad. Sci. U. S. A. 41 (1955), 379-382.

[2] - The principal and chordal principal cluster sets of a certain meromorphic function, Rev. Roum. Math. Pures Appl. 15 (1970), 3-6.

[ 3 ] — G. Piranian and G. S. Young, Intersections of cluster sets, Bul. Inst. Politehn. Iaşi (N.S.) 5 (1959), 29-34.

[4] C. L. Belna, Intersections of arc-cluster sets for meromorphic functions, Nagoya Math. J. 40 (1970), 213-220.

[5] J. T. Gresser, On uniform approximation by rational functions with an application to chordal cluster sets, Nagoya Math J. 34 (1969), 143-148.

[6] A. Hurwitz and R. Courant, Vorlesungen über allgemeine Funktionentheorie und elliptische Funktionen (3d ed.), Berlin, 1929.

[ 7 ] R. Nevanlinna, Eindeutige analytische Funktionen (2d ed.), Berlin, 1953.

University of Wisconsin-Milwaukee 